\title{
Mercados públicos y competitividad: El Palmar y Las Flores de Ciudad Nezahualcóyotl
}

\section{Public markets and the challenge of competitiveness: El Palmar and Las Flores of Ciudad Nezahualcóyotl}

\author{
Laura Elena Garza-Bueno*
}

\begin{abstract}
Recent demographic and economic changes have brought about new ways of acquiring food, which reduce participation of public markets in retail commerce. To counteract this trend, the Mexico State government, in alliance with Colegio de Postgraduados, prepared public market human resources as a strategy to increase competitiveness. El Palmar and Las Flores market stall owners were induced to reflect on their competitive advantages and on those expressed by surveyed clientele. We present the form in which the entire process of reflection developed, results of the survey, actions agreed upon, and thematic contents identified as core elements for future programs.
\end{abstract}

Keywords: food supply, public markets, retail commerce, training.

\section{Resumen}

Con el propósito de modernizar los mercados públicos, el gobierno del Estado de México en alianza con el Colegio de Postgraduados estableció un programa de formación de recursos humanos. La metodología consistió en incorporar a los locatarios de los mercados El Palmar y Las Flores de Ciudad Nezahualcóyotl a un proceso de reflexión sobre las ventajas competitivas que ellos consideran poseer y las que señala su clientela. Estas últimas se obtuvieron mediante una encuesta. El artículo presenta los resultados de la reflexión y de la encuesta, así como de las acciones acordadas a partir de la confrontación de ambos resultados.

Palabras clave: abasto de alimentos, mercado público, comercio minorista, capacitación. 


\section{Introducción}

Los mercados públicos fueron durante mucho tiempo los principales centros de comercialización de productos perecederos como frutas, legumbres, verduras, lácteos, carne, huevo, comida y abarrotes. No obstante, los procesos de urbanización y modernización característicos de los inicios de la segunda mitad del siglo $\mathrm{xx}$, aunados a los cambios estructurales de la economía mexicana de fines de las últimas décadas del mismo periodo, modificaron esa situación. De tal manera que el escenario del comercio al detalle se ha ido transformando para dar lugar a establecimientos comerciales -apoyados en el uso de tecnología, tanto dura como blanda, y el control del acopio y la distribución-, conformando así, un grupo moderno que compite con los establecimientos tradicionales (Bocanegra y Vázquez, 2003). Destacando, en particular, el surgimiento de las tiendas de autoservicio, los centros comerciales y supermercados modernos que se han constituido en una importante competencia para los mercados públicos (Hernández, 2005).

El comercio al detalle del nuevo tipo atiende a partir de un nuevo concepto, el cual se basa en el principio de autoservicio de los clientes, departamentos separados para líneas completas de alimentos y productos no-alimenticios, manejo de grandes volúmenes y precios rebajados para productos específicos. Esta modalidad de comercio minorista presenta la expansión más acelerada. De acuerdo con Schwentesius y Gómez (2006: 208) son el tipo de tienda que durante los años noventa se ha expandido con más dinamismo, al registrar un crecimiento de 499 a 1288 establecimientos entre 1993 y 2000/2001, lo que permite hablar de un proceso de supermercadización en México; ${ }^{1}$ en el que las empresas trasnacionales han participado intensamente (Bocanegra, 2007 y 2008) atraídas por el amplio mercado y las limitadas restricciones impuestas por el gobierno mexicano (Bocanegra y Vázquez, 2003; Abud y González, 2009). El principal resultado de dicho proceso ha sido la transformación del comercio al detalle en México, donde hoy sobresale una importante participación ${ }^{2}$ de los establecimientos comerciales del tipo moderno -tanto de capital nacional, como extranjero- en la captación de ingresos generados por el comercio. ${ }^{3}$

${ }^{1}$ Fenómeno similar al ocurrido en otros países de América Latina (Reardon y Berdegué, 2002).

${ }^{2}$ En 2007, los supermercados generaron el 43. 6\% de la industria (Datamonitor, en Abud y González, 2009: 10).

${ }^{3}$ Situación que no se ve reflejada en una reducción del número de unidades correspondientes al sector tradicional, por el contrario, se ve acompañada de una dinámica participación del comercio tradicional en cuanto a número de unidades y personas ocupadas. Los censos económicos de 1999 y 2004 indican que las tiendas de abarrotes, ultramarinos y misceláneas han crecido $14.6 \%$ en cinco ańos (INEGI, 1999 y 2004) lo que se explica por la relativa facilidad que ofrece el comercio minoris- 
Además de la expansión de las nuevas formas del comercio al detalle cabe destacar el fuerte contraste entre la manera tradicional y la impulsada por las grandes cadenas de minoristas, quienes además de recurrir a fuertes inversiones y hacer uso de las nuevas tecnologías soportan su actividad en el conocimiento de su clientela y sus diversas necesidades, mismas que se han transformado como resultado del, cada vez más alto número de consumidores urbanos, que dispone de menos tiempo para atender las cuestiones domésticas y se ve obligado a realizar largos y tediosos desplazamientos. Lo que junto con la creciente incorporación de la mujer al mercado laboral y el cambio en la composición de las familias -tanto en el papel como en el número de sus miembros- han transformado el comportamiento de los consumidores.

El contraste entre la manera tradicional y moderna del comercio al detalle se confirma al observar numerosos mercados públicos que presentan espacios rústicos y tradicionales con poco mantenimiento; bajo nivel de higiene; locales y pasillos estrechos y en malas condiciones; nula infraestructura para la conservación de perecederos, y sin estacionamiento. A diferencia de los establecimientos modernos como los supermercados, los cuales cuentan con equipos computarizados para el control del ingreso y salida de mercancía y dinero, amplios corredores por donde los consumidores pueden recorrer con la comodidad de un carrito que carga la mercancía deseada, espacios higiénicos, iluminados y ventilados con una amplia gama de productos que permite al consumidor adquirir los productos que necesita sin tener que moverse a distintos establecimientos. Los horarios son a lo largo del día y en algunos supermercados, incluso, las 24 horas. Totalmente un enfoque de comodidad y practicidad para el consumidor (Hernández, 2006a).

En opinión de algunos estudiosos del tema (Duhau y Giglia; 2007: 33), paralela a la supermercadización existe la tendencia del aprovechamiento de las distintas modalidades de comercio minoritario (mercados fijos, tianguis y comercio informal) del que se beneficia un amplio segmento de la población. Tales autores observaron las prácticas de consumo de la población metropolitana en diversas áreas testigo, distribuidas en el Área Metropolitana de la Ciudad de México (АмСм), ${ }^{4}$ con distintos contextos urbanos y socioespaciales, y concluyeron que las alternativas preferidas para la compra de artículos de consumo diario ${ }^{5}$ se distribuyen,

ta para constituirse en una forma de autoempleo que permite amortiguar los efectos de la crisis económica por la que atraviesa el país (Bocanegra y Vázquez, 2003: 22-23).

4 Área Metropolitana de la Ciudad de México, de la cual forma parte Ciudad Nezahualcóyotl, sitio en que se localizan los mercados El Palmar y Las Flores.

${ }^{5}$ Los datos aluden, únicamente, a la primera opción. 
en promedio (entre las 20 áreas del AMCM seleccionadas para el estudio), ${ }^{6}$ de la siguiente manera: $31.8 \%$ tienda de abarrotes; $21.7 \%$ mercados públicos; $9.7 \%$ tianguis; $32.3 \%$ supermercados; $3.2 \%$ otras variantes no especificadas, mientras que $1.4 \%$ sin respuesta. Resultados que ponen en evidencia el significativo porcentaje de la población que sigue recurriendo al mercado público en términos generales y que, en el caso de los estratos bajo a medio-bajo, llega a representar hasta 40\% (Duhau y Giglia, 2007).

En virtud de lo anterior, el Gobierno del Estado de México (GEM, s/f) en alianza con instituciones públicas y privadas, así como los locatarios de los mercados públicos impulsan, desde el 2007, un Programa de Modernización de Mercados, el cual incluye un conjunto de acciones entre las que se incluye la formación de los recursos humanos. En ese contexto, se estableció un compromiso entre el Gobierno del Estado de México y el Colegio de Postgraduados (CP), para diseñar y aplicar un programa de formación de recursos humanos a los comerciantes de diversos mercados públicos.

El Colegio de Postgraduados ${ }^{7}$ es una institución ubicada en el Estado de México que ha tenido una destacada participación interinstitucional a través de numerosos cursos de capacitación en materia de producción, productividad y comercialización de productos agropecuarios, lo que constituyó un importante antecedente para el gobierno estatal.

Los mercados El Palmar y Las Flores de Ciudad Nezahualcóyotl, fueron seleccionados por el Gobierno del Estado de México como los dos primeros en los que se había de llevar a cabo el programa de formación. La principal razón por la que el gobierno estatal los propuso fue la solicitud de un grupo de locatarios para que se les impartiera capacitación para actualizar sus conocimientos. De tal manera que, al mismo tiempo que se satisfacía una demanda, el gobierno estatal podría validar el programa de formación propuesto por el cr. Además de ello, es importante seńalar que, en el momento en que se signó el acuerdo GEM-CP, los locatarios experimentaban una serie preocupación por la, entonces, próxima inauguración de una tienda de la cadena Walmart a unas cuantas cuadras del mercado. Ya que, aun cuando existían otros supermercados cercanos (Aurrera y Gigante), ninguno de ellos se ubicaba con tanta proximidad ni eran considerados tan fuertes competidores.

\footnotetext{
${ }^{6}$ Las áreas seleccionadas por los autores incluyen población con ingresos clasificados como bajos y muy bajos; medios; medio bajo y medio alto y alto.

El Colegio de Postgraduados está adscrito a la Secretaría de Agricultura, Ganadería, Desarrollo Rural, Pesca y Alimentación (Sagarpa). Desde su creación, hace más de cincuenta años, el cP está dedicado a la docencia, investigación y servicio. Destacando, en particular, que el cr cuenta con una larga experiencia en materia de vinculación con empresas e instituciones estrechamente ligadas al sector rural.
} 
Los mercados El Palmar y Las Flores se localizan en Ciudad Nezáhualcóyotl, ${ }^{8}$ municipio del Estado de México (mapa anexo). Ambos mercados ocupan una misma manzana aunque corresponden a dos diferentes colonias, es decir, uno está orientado hacia la colonia El Palmar y, el otro, hacia la de Las Flores. Tanto la superficie, como el número de locales de que disponen cada uno de los mercados son muy similares: El Palmar cuenta con una superficie de $1,350 \mathrm{~m}^{2}$, y 153 locales, mientras que Las Flores tiene una superficie de $1,500 \mathrm{~m}^{2}$ y 148 locales. Ambos mercados se encuentran físicamente integrados, sus construcciones son contiguas al grado de confundir materialmente sus límites, lo que de alguna manera representa una imagen de un gran mercado. Sin embargo, cada uno tiene su propia administración, es decir, dos organizaciones directivas, así que aun cuando comparten un mismo espacio e infraestructura son administrativamente independientes.

El compromiso de llevar a cabo un programa de formación entre los locatarios de dichos mercados significó un importante reto para el Colegio de Postgraduados y para quien suscribe el presente texto como responsable directa del mismo; primero, porque las expectativas del GEM se enfocaron en detonar procesos que condujeran realmente a la modernización del mercado público más que en acumular horas de capacitación, es decir, se trataba de impulsar cambios en la realidad y no sólo de explicarlos; segundo, porque el enfoque de la capacitación que el cP promueve desde hace algunos ańos (Garza, 2006), requiere una didáctica especializada que obliga a un diagnóstico del binomio conformado por el sujeto o actor social en cuestión y la unidad en que desarrolla su actividad económica, en este caso locatarios/mercado público, binomio que no había sido abordado en ninguna de las experiencias anteriores del equipo del cr. Bajo ese contexto, El Palmar y Las Flores se volvieron los mercados piloto donde se habría de probar un nuevo modelo de formación, el cual podría extenderse (como sucedió en la realidad) a otros mercados públicos del Estado de México.

El diagnóstico del mercado público obligó a una búsqueda de información con pocos resultados. De tal manera que la escasa información sobre la situación actual del mercado público obligó a un esfuerzo inquisitivo que involucró a académicos y estudiantes de diversas instituciones, a un grupo de profesores del CP, a los locatarios y diversos analistas del área de comercio del GEM. Sin el compromiso asumido por todos ellos,

\footnotetext{
${ }^{8}$ El municipio de Nezahualcóyotl está ubicado en la periferia oriente de la ciudad de México es uno de los de mayores dimensiones del Estado de México; es parte, también, de los 10 municipios más grandes del país, ya que ocupa el noveno lugar pues cuenta con 1.1 millones de residentes (Conapo, 2005).
} 
los limitados recursos de que se disponía para la capacitación no hubieran permitido los alcances de la investigación que aquí se presenta. ${ }^{9}$

La propuesta de formación del CP parte de reconocer que el diagnóstico de la unidad económica hecho al margen de los sujetos sociales involucrados, rara vez conduce a proyecciones de la realidad que se pongan en práctica. De ahí que se planteara la aplicación de la metodología denominada trabajo-aprendizaje ( $\mathrm{t}-\mathrm{a})$, la cual toma como punto de partida que los procesos de formación deben constituir situaciones de reflexión de las propias condiciones y procesos de trabajo, ${ }^{10}$ que propicien cambios en las percepciones de los individuos, incitándolos a visualizar acciones que les permitan mejorar su actividad económica.

En concordancia con lo anterior, el equipo del CP se planteó un conjunto de tareas que -en el mismo orden en que se enlistan- presentan la estructura del presente documento. La primera consistió en identificar las ventajas competitivas del servicio de comercio al detalle, como un ejercicio de conceptualización que posteriormente se compartió con los locatarios de los mercados El Palmar y Las Flores para iniciar la reflexión sobre las preferencias de consumo en la actualidad y la manera en que el comercio minorista ha respondido a las mismas. La segunda consistió en establecer, conjuntamente con los comerciantes, las debilidades y fortalezas de los mercados El Palmar y Las Flores, lo que derivó, sobretodo, en el compromiso de profundizar en el conocimiento de su clientela. La tercera, se fundamentó en la realización de una encuesta a 279 clientes, junto con el análisis de sus resultados llevado a cabo con los comerciantes, el cual sirvió de base para ejecutar proyectos de acción por parte de los locatarios, así como para el diseño de programas de formación dirigidos a locatarios de otros mercados públicos.

La riqueza de la información obtenida frente a la urgente necesidad de identificar los conocimientos estratégicos para la mejor inserción de los mercados públicos en el comercio al detalle, motivaron la elaboración del presente documento.

\footnotetext{
${ }^{9}$ En particular queremos destacar la participación del doctor Oscar Figueroa R. del cP; del maestro en ciencias J. Bernardo Solís de la UACH; del maestro en ciencias Erick Quesnel G.; de las capacitadoras: licenciada Adriana Moreno D. y licenciada Eva Clara Geronés; de los representantes de ambos mercados: Alberto Alvarado y Alfonso Ortega, y de la colaboración de la maestra en ciencias Ana Ma. Durán C. y del licenciado Martín Durán C. de la UAM-Azcapotzalco. Participación que fue posible gracias a la confianza que los responsables del área de comercio del GEM, el médico veterinario zootecnista José Antonio Ordoñez R. y el ingeniero Víctor Ramírez M. mantuvieron en todo momento en un ejercicio de formación poco convencional.

${ }^{10}$ Para mayor información sobre el método trabajo-aprendizaje (véase Duch et al., 2005 y Duch, 2007).
} 


\section{La competitividad de los mercados públicos. Aspectos conceptuales}

Dado que el interés central del programa de formación está en mejorar la posición de los mercados públicos en el comercio al detalle, es necesario recurrir al concepto de competitividad, en tanto, de ésta depende la inserción y el reposicionamiento, así como la permanencia de productos y servicios en el gusto de la clientela.

En virtud de lo anterior, una de las primeras tareas consistió en precisar el concepto de competitividad para el caso de los servicios otorgados por el mercado público, para ello, se partió de una visión sencilla y ampliamente conocida del concepto, la competitividad es la capacidad de competir en los mercados de bienes o servicios. Dicha capacidad depende de una multiplicidad de factores. Sin embargo, para Porter (1999: 21-22), "La ventaja competitiva de una empresa nace fundamentalmente del valor que una empresa es capaz de crear para sus compradores, que exceda el costo de esa empresa por crearlo". Es decir que, para alcanzar y mantener su ventaja, las empresas deben ofrecer productos y servicios que representen -para el comprador- un valor mayor a su costo. Valor que está en función de la satisfacción que experimenten no sólo de sus necesidades, sino también de sus deseos (Kotler y Armstrong 1998; 2001).

La satisfacción de necesidades aparejada a la de deseos es un tema abordado por los estudiosos del comportamiento del consumidor, quienes establecen que la visión tradicional, parte de dos ideas propagadas por los economistas: el postulado de la racionalidad y la restricción presupuestaria de la conducta del consumidor. Ideas que se reconocen como una parte importante de la hipotética función del consumo, pero que no pueden explicar por sí solas todas las diferencias que se observan en los consumidores. Razón por la cual recurren a otras disciplinas como la psicología y la sociología (Alonso y Grande, 2004; Mollá et al., 2006). La diversidad de enfoques que existen, tanto en la ciencia económica como en la psicología y la sociología y las distintas maneras de interrelacionar los aportes de cada una de ellas, dificultan la selección de un esquema único de la estructura de los procesos de toma de decisiones del consumidor. Sin embargo, muestran un denominador común: el ser humano está influido por fuerzas internas que moldean su conducta, así también, por fuerzas externas como el comportamiento social (Alonso y Grande, 2004; Mollá et al., 2006; Rivera et al., 2000).

En virtud de lo anterior, la aplicación del concepto de competitividad adoptado obliga, tanto a la revisión de los precios con respecto a la competencia como al análisis de la visión de calidad, que los mismos consumidores tienen de este tipo de comercio (Kinnear y Taylor, 1996). 
A partir de los planteamientos anteriores y del compromiso de capacitación se tomaron las siguientes decisiones: respecto a la variable precio, se acordó analizarla a través de las opiniones de los locatarios y de la percepción de sus clientes, esto debido a que una investigación basada en fuentes primarias excedía las posibilidades del equipo (académicos y locatarios), además de que los conocimientos de los locatarios sobre los precios de sus competidores parecieron suficientes.

En cuanto a la calidad, se optó por identificar un conjunto de variables, algunas de ellas utilizadas para valorar los servicios de comercio al detalle que ofrecen los supermercados (Kotler y Armstrong 1998; ACNielsen, 2006); así como otras encontradas en documentos alusivos a la problemática de las centrales de abasto (Secretaría de Economía, 2008) y de los mercados públicos (Hernández, 2005 y 2006a y 2006b), y otras propuestas a partir de lo observado. Todo ello, permitió establecer la lista de las variables base para evaluar la competitividad de los mercados públicos, a la luz de sus comerciantes y de su clientela. El resultado fue el siguiente:

1) Calidad del producto, para el caso de perecederos: fresco, pertinente (manera en que el cliente lo pide); compras en pequeñas proporciones, combinaciones de productos, distintos grados de madurez para un mismo producto, productos frescos que se dejan listos para ser utilizados.

2) Diversidad de productos: posibilidad de encontrar todos los productos que se requieren en el hogar.

3) Servicio al cliente: amable, rápido, honesto.

4) Familiaridad: conocimiento del cliente, relación de lealtad entre el cliente y el comerciante.

5) Practicidad: ubicación, horarios, tarjetas de crédito y débito, vales de despensa.

6) Financiamiento: crédito.

7) Calidad del lugar: seguridad, limpieza, iluminación, ordenamiento, espacio.

A partir de la identificación de las variables anteriores por parte del equipo del cr se procedió a hacer un ejercicio que, mediante la exposición de las dichas variables, permitiera ubicar la visión de los locatarios respecto a la calidad de sus servicios y, por supuesto, a sus ventajas competitivas. Los resultados del mismo se comentan en el siguiente punto. 


\section{La detección de la necesidad de conocer a sus clientes}

Con el propósito de avanzar en el proceso de identificación, por parte de los locatarios, de los aspectos que limitan o posibilitan la competitividad de los mercados públicos El Palmar y Las Flores se realizó el ejercicio denominado FODA (Fortalezas/Oportunidades y Debilidades/Amenazas). Esta herramienta analítica facilita sistematizar la información que la organización (representada, en este caso, por los locatarios) tiene sobre el mercado y sus variables. Este tipo de análisis tiene la ventaja de combinar los aspectos internos a través de las fortalezas y debilidades con las fuerzas externas que se identifican como oportunidades y amenazas.

Para llevar a cabo el ejercicio antes señalado se determinó realizar las reuniones de diagnóstico con tres subgrupos (organizados por giros), de tal manera que se pudiera abarcar el universo completo de 268 locatarios y 76 giros. En los hechos, las reuniones de diagnóstico contaron con la asistencia únicamente de 70 personas, ${ }^{11}$ (representantes de 35 giros) que atendieron al llamado de acuerdo con sus tiempos libres, lo que motivó heterogeneidad de giros en cada subgrupo. Situación que, aunque no estaba prevista, no representó mayor problema para la realización del ejercicio.

Una presentación esquemática de los principales resultados alcanzados en el total de las sesiones dedicadas al FODA, se puede observar en el cuadro 1, el cual contiene las Fortalezas y Debilidades, así como las Oportunidades y Amenazas que mencionaron los comerciantes y que, sirvieron de base para planear acciones a futuro. Respecto al cuadro: las filas siguen el mismo orden de las variables que determinan la competitividad, las cuales aparecen enlistadas en el punto anterior. Ordenamiento que sirvió para soportar los planes de acción y la organización de los contenidos de la formación. Destacando, por último, que la redacción del cuadro respeta los textos redactados conjuntamente con los locatarios.

Una vez concluido el ejercicio FODA, las acciones de planeación estratégica que se acordaron entre los locatarios no alcanzaron la envergadura que se esperaba por parte del equipo del cr. Sin embargo, permitieron la implementación de un conjunto de acciones tendientes a favorecer sus fortalezas y superar sus debilidades, tales como la organización (de una parte) de los comerciantes para impulsar publicidad como volantes, lonas, etc., que les permitiera atraer más clientela; gestionar apoyos gubernamentales para mejorar su infraestructura; dialogar con los responsables de los bicitaxis para incitarlos a mejorar sus servicios y, otras acciones

\footnotetext{
${ }^{11}$ El resto también se atendió pero con otra estrategia: mediante la difusión de los resultados de la encuesta y el contraste de éstos con su punto de vista.
} 


\section{Cuadro 1 \\ Fortalezas, Debilidades, Oportunidades y Amenazas}

\begin{tabular}{l} 
Fortalezas \\
\hline Precios razonables \\
Frescura \\
Variedad de giros con buen surtido \\
Atención personalizada, buen trato \\
al cliente, respeto, atención, gentileza \\
Tradición, conocen al cliente \\
La ubicación y que algunos comerciantes \\
empiezan a recibir tarjetas de crédito y \\
débito, estacionamiento \\
Crédito, sistema de apartado y regateo \\
Limpieza, la luz no falla, existe vigilancia, \\
recolección de basura diariamente
\end{tabular}

Falta de orden en la distribución de los giros Conocen poco a sus clientes y se tiene conocimiento de casos de abuso del cliente

Consejos culinarios y recetas, entrega a domicilio, cursos (elaboración alimentos), servicio a la comunidad; degustaciones Organización, compañerismo, unión

Falta de comunicación entre locatarios, egoísmo, resistencia al cambio; falta de apego al reglamento

\begin{tabular}{ll}
\hline \multicolumn{1}{c}{ Oportunidades } & \multicolumn{1}{c}{ Amenazas } \\
\hline $\begin{array}{l}\text { Potencial económico por crecimiento de la } \\
\text { zona }\end{array}$ & $\begin{array}{l}\text { Las grandes cadenas comerciales (de próxi- } \\
\text { ma inauguración) }\end{array}$ \\
$\begin{array}{ll}\text { Construcción de nuevas unidades habita- } \\
\text { cionales }\end{array}$ & El comercio informal \\
& $\begin{array}{l}\text { El mal servicio de los bicitaxis que trasladan } \\
\text { a numerosos clientes }\end{array}$ \\
\hline
\end{tabular}

Fuente: Elaboración propia con base en la información obtenida en los talleres del Programa de Formación GEM-CP.

menores. Pero, sobretodo, despertaron la inquietud, prácticamente generalizada, respecto a la certidumbre de que conocían a su clientela. ${ }^{12}$

Sin duda, una de las acciones estratégicas más relevantes -acordadas por el grupo de capacitadores y capacitados- fue la de profundizar en el

${ }^{12}$ La pregunta generadora de la duda fue: ¿Cuántos clientes visitan el mercado diariamente?, la respuesta más frecuente fue de 500 personas al día, aunque reconocieron ignorar el origen de ese dato. Ante la falta de información confiable al respecto se propuso realizar un conteo. Los resultados del mismo sorprendieron a todos: 3974 clientes ingresaron un solo día. Cifra que representa cerca de $800 \%$ más del número manejado por los locatarios. Cabe señalar que el conteo se hizo en forma cuidadosa, entre otras cosas, porque no todos los visitantes son compradores, dado que los mercados se encuentran localizados en una manzana bastante amplia, los pasillos de los mismos son una manera de acortar su camino; de ahí que se optara por preguntar si la visita era con fines de consumo. La segunda observación es que en el conteo de compradores se realizó de manera simultánea en las ocho puertas de que dispone el mercado, con miembros del equipo del CP, estudiantes de diferentes instituciones y algunos locatarios. 
conocimiento del cliente (primer paso para mejorar la competitividad). Ahora, ese reconocimiento puso de manifiesto la necesidad de realizar una encuesta para conocer el perfil del cliente que acude a El Palmar y Las Flores, así como sus preferencias. Lo que, además de brindar más información con respecto a los compradores, permitiría contrastar las ventajas competitivas, observadas por los comerciantes, y las que visualiza la clientela.

\section{La estrategia para conocer mejor al cliente}

\subsection{Diseño de la encuesta}

A fin de que los locatarios adquirieran mayores y mejores conocimientos sobre su clientela se diseñó y aplicó una encuesta entre los clientes que acuden al mercado (ECAM de aquí en adelante). Para el diseño del cuestionario se realizó una lista de preguntas que fue puesta a consideración de los locatarios, quienes la revisaron e incluyeron preguntas de su interés. A partir de dicha revisión se formuló el primer borrador del cuestionario, el cual fue sometido a una prueba piloto.

El cuestionario final dirigido a los clientes de El Palmar y Las Flores incluyó 65 preguntas, de las cuales 40 son cerradas y 25 son cuestionamientos abiertos, todas ellas contenidas en cuatro apartados: el primero, destinado a conocer el perfil del comprador; el segundo apartado se formuló con el objeto de conocer las características del consumo que habitualmente realiza el cliente (sea o no en el mercado); el siguiente punto fue expresamente diseñado para conocer las características del consumo que el cliente realiza en El Palmar y Las Flores; por último, se incluyó un apartado que concentrara las opiniones de los clientes sobre los artículos y servicios que brindan ambos mercados.

\subsection{Diseño de muestra}

Previo al levantamiento de la encuesta se determinó la muestra mediante la siguiente fórmula:

$$
\mathrm{n}=\frac{\mathrm{N}^{*} \mathrm{p}^{*} \mathrm{q}}{(\mathrm{N}-1) / \mathrm{z} 2^{*} \mathrm{~d} 2^{*} \mathrm{p} 2+\mathrm{p}^{*} \mathrm{q}}
$$

Con un grado de confiabilidad de $95 \%(Z=1.96)$ y un porcentaje de error de $10 \%(\mathrm{~d}=0.10)$. Como resultado se obtuvo un tamaño de muestra de 254 para el universo total. 


\subsection{Levantamiento y captura de la información}

Se aplicaron 279 cuestionarios, 25 más de lo previsto, con una importante participación de los locatarios en el levantamiento de la encuesta. Una vez concluida la captura de todas las encuestas en las bases de datos, la información fue transferida a Excel, para finalmente transformarse en bases de datos en SPss (Statistical Package for Social Sciences) que fue el paquete estadístico utilizado para procesar toda la información capturada.

\subsection{El consumidor y sus preferencias (Resultados de la encuesta)}

\subsubsection{Perfil del cliente}

De acuerdo con lo esperado, la población que acude al mercado está constituida, en su mayoría, por mujeres. No obstante, los porcentajes no correspondieron a lo esperado por los locatarios: el número de hombres superó sus previsiones. De acuerdo con los resultados obtenidos: $82.8 \%$ son mujeres y $17.2 \%$ son hombres. El promedio de edad es de 41.5 años, aunque la desviación estándar es elevada, es decir, que a los mercados acuden compradores de prácticamente todas las edades (figura I).

Figura I

\section{Edad de las personas encuestadas}

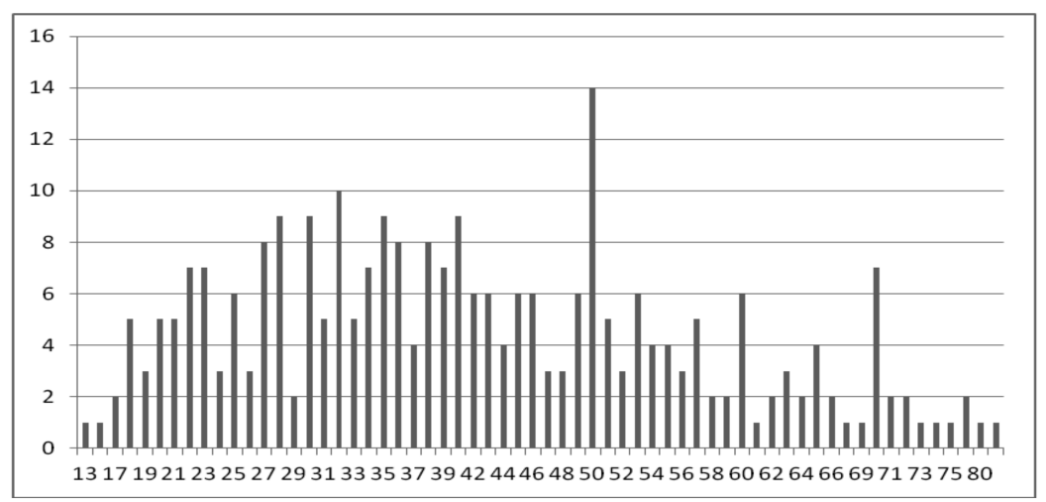

Fuente: Elaboración propia con base en la ECAM.

En cuanto al medio de acceso al mercado, los datos confirmaron algo sabido: la mayoría de los clientes (82.4\%) llega caminando, y/o en transporte público (13.6\%). Sin embargo, sorprendió a los comerciantes el bajo número de clientes que llegan en automóvil (2.5\%), pues ellos suponían una cifra mucho mayor. El resto $(1.5 \%)$ incluye otros medios de transporte propios. 
Con respecto al horario en que la clientela acude al mercado, la encuesta mostró que poco más de $70 \%$ acude entre las 10 y las 15 horas. El resto $(29.1 \%)$ se distribuye en el resto del día.

En relación con los ingresos, la población encuestada se ubicó en tres rangos de ingreso mensual: la mayoría (53\%) obtiene ingresos que fluctúan entre 1,500 y 4,500 pesos. El resto $21.5 \%$ percibe menos de 1,500 pesos y, 21.9\% manifestó que sus ingresos son superiores a los 4,500 pesos. La diferencia está conformada por personas que desconocen el dato o se negaron a contestar.

\subsubsection{Caracteristicas generales del consumo}

En atención a la reconfiguración de los hábitos de consumo planteadas por los especialistas en el tema, se consideró primordial conocer la manera en que éste se ha reestructurado entre la población que nos ocupa. De esta manera, los comerciantes podrían confirmar o rechazar sus supuestos al respecto.

Derivado de lo anterior, la primera pregunta tuvo el fin de conocer la frecuencia con que los clientes acuden a los mercados El Palmar y Las Flores. El resultado muestra que la mayor parte de la clientela lo visita diariamente o con mucha frecuencia: $65.2 \%$ diariamente; $13.3 \%$ de tres a cinco veces por semana; $17.9 \%$ de una a dos veces por semana. De tal manera que apenas $3.6 \%$ va dos veces por mes o de manera ocasional. Una posible explicación a la elevada frecuencia de las visitas es la cercanía de las escuelas, ya que los actos de consumo están fuertemente influidos por la movilidad propia de los compradores. Explicación que se fortalece si también se observan los horarios de mayor afluencia.

Con relación a los supermercados, las personas encuestadas manifestaron ser poco asiduas a visitar el supermercado o, de plano, no acudir nunca a ese tipo de establecimientos: $40.1 \%$ afirmó ir ocasionalmente o cerca de una vez al mes, mientras que $27.6 \%$ dijo que no iba nunca; el resto, $32.3 \%$, se distribuye de la siguiente manera: $17.9 \%$ va una vez a la quincena; $10 \%$ de una a dos veces por semana y, 3.9\% más de tres veces por semana.

Una situación diferente se manifestó con respecto al tianguis. Sobre este tipo de comercio al detalle más de la mitad de la población encuestada señaló que acude al tianguis con frecuencia: $54.1 \%$ lo hace de una a dos veces por semana y $4.7 \%$ lo visita tres veces o más a la semana. $\mathrm{Al}$ respecto, es importante señalar que un tianguis se establece cada miércoles sobre la 4a Avenida, frente a los mercados El Palmar y Las Flores, lo que hace pensar que acuden a ese en particular porque queda de paso por las escuelas. La otra parte de los encuestados (41.2\%) se comporta 
de la siguiente manera: $5.7 \%$ una vez al mes; $12.2 \%$ una vez al mes y $23.3 \%$ nunca.

Adicionalmente, se incluyó una pregunta con el fin de saber en qué otros lugares suelen comprar los clientes además del tianguis y, eventualmente, en los supermercados. Las respuestas obtenidas indican que $58.3 \%$ no compra en otros lugares, mientras que $41.7 \%$, sí lo hace. Para complementar esta información se les preguntó a las 116 personas que afirmaron que compraban en otros lugares, además del tianguis y el supermercado, ¿cuál era ese lugar?, la respuesta -en 97 de los 116 casos señalados- fue la tienda de abarrotes. Mientras que las otras modalidades de comercio al detalle como el ambulante, el de ventanilla o a domicilio, no presentan la misma importancia ya que apenas unas 20 personas manifestaron utilizar este tipo de comercio, de las cuales, la mayoría menciona esta opción conjuntamente con la tienda de abarrotes.

La comparación entre las visitas al mercado público y las que se realizan en otras modalidades de comercio minorista, indica que la población encuestada prefiere el mercado para adquirir artículos de consumo diario, y coloca en segundo término al tianguis, seguido del supermercado. La explicación a este orden de preferencia seguramente está relacionada con los niveles de ingreso de la clientela y la movilidad.

A fin de conocer más la apreciación con respecto al comercio minorista que compite con el mercado público se solicitó a las personas encuestadas que señalaran las ventajas y desventajas que encuentran en ellas, las respuestas que se obtuvieron con mayor frecuencia, primero para el tianguis y después para el caso de los supermercados, se presentan en los cuadros 2 y 3 .

\section{Cuadro 2 Ventajas y desventajas del tianguis}

\begin{tabular}{lclc}
\hline \multicolumn{1}{c}{ Ventaja } & Frecuencia & \multicolumn{1}{c}{ Desventaja } & Frecuencia \\
\hline Precio (bajo) & 127 & No pesan bien & 80 \\
Surtido (productos) & 20 & Calidad (baja) & 30 \\
Ubicación (cercanía) & 19 & Ninguna & 19 \\
Ninguna & 12 & Precio (alto) & 10 \\
\hline
\end{tabular}

Fuente: Elaboración propia con base en la ECAM.

Los resultados llaman mucho la atención, pues la principal opinión favorable al tianguis es que el precio se considera bajo; opinión que fue señalada por cerca de la mitad de los 279 encuestados, sin embargo, la desventaja que aparece en primer lugar -también con una elevada frecuencia- es que "no pesan bien", es decir que les parece más barato aún 
cuando se saben engañados. El hecho concreto para los locatarios de El Palmar y Las Flores es que los miércoles se coloca un tianguis, en la cercanía de sus instalaciones, lo que les provoca una notoria baja en sus ventas a causa de los, aparentemente, menores precios que ofrece.

\section{Cuadro 3}

Ventajas y desventajas del supermercado

\begin{tabular}{|c|c|c|c|}
\hline Ventaja & Frecuencia & Desventaja & Frecuencia \\
\hline Surtido y variedad & 40 & Caro & 61 \\
\hline Ofertas & 37 & Distancia (lejanía) & 38 \\
\hline $\begin{array}{l}\text { Barato (en general o en casos } \\
\text { particulares) }\end{array}$ & 35 & $\begin{array}{l}\text { Menor calidad (verduras, } \\
\text { carnes) }\end{array}$ & 21 \\
\hline $\begin{array}{l}\text { Instalaciones (limpieza, clima, } \\
\text { tamańo, orden) }\end{array}$ & 19 & Ninguna & 15 \\
\hline Ninguna & 9 & & \\
\hline
\end{tabular}

Fuente: Elaboración propia con base en la ECAM.

Con respecto a la información plasmada en el cuadro 3 se destaca, entre las ventajas, el número de respuestas que mencionan las ofertas y el hecho de que es más barato, cifra que supera, al observarse de manera conjunta, al de las que señalan la diversidad (surtido y variedad). Lo que hace suponer que la principal ventaja es, en realidad, el bajo precio que manejan los supermercados en algunos de sus productos. Con menor frecuencia, los encuestados señalaron aspectos como calidad, condiciones de las instalaciones, horarios y vales de despensa. Con respecto a la variable horario, hay que tener presente que la mayoría de las personas encuestadas fueron amas de casa, para quienes esta variable en el servicio no es relevante.

En lo correspondiente a las desventajas de los supermercados destaca como la principal variable: el precio, es decir, que consideran que el supermercado vende caro. Esta situación, en contradicción con opiniones de otros encuestados que piensan que el supermercado es barato, nos lleva a suponer que los productos que, de manera específica, atraen al consumidor los de menor precio a los del mercado público, aunque el resto de los artículos que ofrece el mismo supermercado le parezcan al comprador más caros. Este supuesto, parte del hecho de que los supermercados, dadas sus economías de escala y otras prácticas (como compartir costos con sus proveedores), los lleva a ofrecer numerosos productos a mejores precios que los que se obtienen en otros comercios minoristas.

Con respecto a las tiendas de abarrotes, la opinión de las personas encuestadas está focalizada: de las 107 personas que acuden a las tiendas 
de abarrotes, 83 señalaron hacerlo por la cercanía; cualquier otro tipo de afirmación presenta frecuencias mínimas. Destaca el hecho de que asisten a dichos comercios a pesar de que saben que es caro y con poco surtido (59 y 12 afirmaciones, respectivamente), aunque están conscientes de que representan la solución a necesidades imprevistas o, por las que no vale la pena desplazarse más lejos.

\subsubsection{Características del consumo en los mercados El Palmar y Las Flores}

Con el propósito de particularizar en el caso específico de los mercados que nos ocupan se solicitó a la clientela encuestada señalar los cinco principales productos que acostumbra comprar en El Palmar y Las Flores. El resultado obtenido para cada una de las cinco variantes se esquematiza en el cuadro 4, donde se pone en evidencia la contundente importancia de las frutas y verduras.

\section{Cuadro 4 \\ Principales productos que acostumbra comprar}

\begin{tabular}{lcc}
\hline \multicolumn{1}{c}{ Productos } & $\begin{array}{c}\text { Número de personas que } \\
\text { acostumbran comprar ese tipo } \\
\text { de productos }\end{array}$ & $\begin{array}{c}\text { Porcentaje } \\
\text { (respecto al total de los } \\
\text { 279 encuestados) }\end{array}$ \\
\hline Frutas y verduras & 271 & 97.1 \\
Carnes & 242 & 86.7 \\
Pescado y mariscos & 161 & 57.7 \\
Abarrotes & 108 & 38.7 \\
Lácteos y carnes frías & 73 & 26.2 \\
\hline
\end{tabular}

Fuente: Elaboración propia con base en la ECAM.

Tomando en cuenta que una de las variables que determinan la competitividad de los productos es la disponibilidad de éstos, se preguntó a la población de la muestra si consideraba que el mercado tenía los productos necesarios; la respuesta fue afirmativa en $88.5 \%$ de los casos. Solamente $3.9 \%$ de los encuestados dijeron que no; el resto $(7.5 \%)$ no contestó la pregunta.

Una pregunta de fundamental importancia para este apartado se refiere a las razones por las que los clientes prefieren los productos que compran en El Palmar y Las Flores. Aunque las respuestas contenidas en la encuesta aluden a todos los productos que el encuestado mencionó, el cuadro 1 se refiere, exclusivamente, a los catalogados como principales: i) frutas y verduras; ii) carnes; iii) pescado y mariscos; iv) abarrotes y, v) lácteos y carnes frías. Destaca que en todos los casos, la principal razón 
fue la calidad; la segunda, la pertinencia (es lo que necesito) y, en tercer lugar, el precio.

\section{Cuadro 5 \\ Razones por las que prefiere comprar en el mercado los artículos de su preferencia}

\begin{tabular}{lccccc}
\hline Razones & $\begin{array}{c}\text { Frutasy } \\
\text { verduras } \\
\%\end{array}$ & $\begin{array}{c}\text { Carnes } \\
\%\end{array}$ & $\begin{array}{c}\text { Pescadoy } \\
\text { mariscos } \\
\%\end{array}$ & $\begin{array}{c}\text { Abarrotes } \\
\%\end{array}$ & $\begin{array}{c}\text { Lácteos y/o } \\
\text { carnes frías } \\
\%\end{array}$ \\
\hline Precio & 25.0 & 18.2 & 14.6 & 24.0 & 17.2 \\
Calidad & 42.7 & 50.2 & 32.3 & 16.5 & 25.8 \\
Es lo que necesito & 30.1 & 19.4 & 15.4 & 21.5 & 21.1 \\
No corresponde & 1.8 & 11.8 & 37.3 & 37.6 & 35.5 \\
No contestó & 0.4 & 0.4 & 0.4 & 0.4 & 0.4 \\
Total & 100.0 & 100.0 & 100.0 & 100.0 & 100.0 \\
\hline
\end{tabular}

Fuente: Elaboración propia con base en la ECAM.

\subsubsection{Opiniones de la clientela}

El último apartado de la encuesta se enfocó en conocer las opiniones de los compradores con respecto a las variables: servicio al cliente; familiaridad; practicidad; calidad del lugar y servicios adicionales; por tanto, la primera pregunta del cuestionario estuvo dirigida a conocer su percepción con respecto a la atención. El resultado muestra que poco más de la mitad de la población encuestada (56\%) considera buena la atención brindada, cerca de una cuarta parte (24\%) opina que es muy buena y $18 \%$ la califica como regular. El resto piensa que es mala o, simplemente, no opinó.

La siguiente pregunta de este apartado de la encuesta se formuló con el propósito de averiguar el grado de confianza que la clientela tiene en los comerciantes de los mercados El Palmar y Las Flores. El resultado muestra que menos de la mitad $(43.7 \%)$ de las personas encuestadas tienen mucha confianza en los locatarios, mientras que $45.2 \%$, cercano a la mitad, calificó su grado de confianza como regular; el resto indicó que poca o ninguna o, no contestó. La principal razón que dieron para justificar su grado de confianza fue el tiempo de conocer al locatario; otra importante razón fue el conocimiento que tienen del servicio que les brindan.

El interés de los locatarios en conocer más a fondo la opinión de sus clientes llevó a incluir en el cuestionario una serie de preguntas sobre la calidad del lugar: seguridad, limpieza, baños y locales. El resumen de las respuestas se presenta en el cuadro 6 , donde se puede observar que las 
apreciaciones de regular y malo, aun cuando no superan a las de bueno y regular, no dejan de ser muy significativas.

\section{Cuadro 6}

¿Cómo califica los servicios de seguridad, limpieza, baños y locales?

\begin{tabular}{lcccc}
\hline Calificación & $\begin{array}{c}\text { Seguridad } \\
\%\end{array}$ & $\begin{array}{c}\text { Limpieza } \\
\%\end{array}$ & $\begin{array}{c}\text { Baños } \\
\%\end{array}$ & $\begin{array}{c}\text { Locales } \\
\%\end{array}$ \\
\hline Muy bueno & 15.4 & 12.5 & 8.6 & 12.5 \\
Bueno & 53.8 & 42.3 & 34.4 & 55.2 \\
Regular & 26.9 & 39.1 & 29.0 & 30.1 \\
Malo & 2.5 & 5.4 & 5.7 & 1.1 \\
No contestó & 1.4 & 0.7 & 22.2 & 1.1 \\
Total & 100.0 & 100.0 & 99.9 & 100.0 \\
\hline
\end{tabular}

Fuente: Elaboración propia con base en la ECAM.

Las dos últimas preguntas del cuestionario pretenden conocer lo que más y lo que menos le gusta a los clientes encuestados de El Palmar y Las Flores; los resultados (cuadro 7) muestran que las dos respuestas más frecuentes fueron el surtido y la atención, lo que coincide plenamente con las fortalezas detectadas. En el caso contrario, lo que no les gusta, la respuesta predominante fue la apariencia (imagen/higiene), asunto que aunque fue mencionado entre las debilidades y no coincide totalmente con las deficiencias mencionadas por la clientela. Ya que los locatarios visualizaron más las deficiencias en la infraestructura que en aspectos relacionados con la higiene como la presencia de los perros.

\section{Cuadro 7}

\section{Lo que más le gusta y lo que menos le gusta del mercado}

\begin{tabular}{lclc}
\hline \multicolumn{1}{c}{ Opiniones } & Frecuencia & \multicolumn{1}{c}{ Opiniones } & Frecuencia \\
\hline Surtido (variedad productos) & 92 & $\begin{array}{l}\text { Apariencia, imagen, higiene } \\
\text { (limpieza, olor, drenaje, basu- }\end{array}$ & 86 \\
ra, perros) & 85 \\
Atención (trato, relación) & 68 & Nada & 23 \\
Todo & 35 & Instalaciones (incómodas) & 18 \\
Ubicación (cerca) & 25 & Servicio-atención (deficiente) & 14 \\
Otros & 19 & Seguridad (baja) & 14 \\
\hline
\end{tabular}

Fuente: Elaboración propia con base en la ECAM. 


\section{Conclusiones}

Los resultados de la encuesta aplicada a los clientes que acuden a los mercados El Palmar y Las Flores indican que-como era de esperarse-sus hábitos de compra se distribuyen entre todas las modalidades de comercio minorista. No obstante, su recurrencia los servicios de comercio muestra una marcada preferencia hacia el mercado público y el tianguis.

Asimismo, la encuesta puso en evidencia que la principal ventaja competitiva de ambos establecimientos es la calidad, entendida ésta, fundamentalmente, como frescura de los perecederos. La ventaja competitiva que aparece en segundo lugar es la pertinencia (es lo que necesito), es decir, que las personas encuestadas valoran primordialmente que el producto que adquieran tenga las características requeridas, las cuales pueden ser compras fraccionadas o combinadas (de verduras o carnes); pequeñas cantidades; presentación específica (de carnes o verduras).

Además de lo anterior, la encuesta mostró que los interrogados colocan en un tercer lugar a la ventaja competitiva precio, sin embargo, la interpretación de las opiniones debe tener en cuenta la combinación calidad/ precio. Esto significa que los locatarios seguirán siendo competitivos si sus precios (en los productos señalados) siguen siendo tan bajos, o más, que los de sus competidores. En caso contrario, la clientela puede optar por otra combinación.

El servicio al cliente y la familiaridad pueden ser considerados como ventajas competitivas que se ubican en cuarto lugar, esto es como resultado de la elevada apreciación del cliente. La diversidad puede ser considerada en el quinto lugar de las ventajas competitivas valoradas, sin embargo, es muy probable que la diversidad señalada comprenda, básicamente, los tres tipos de productos que prioritariamente la gente acostumbra comprar (frutas, verduras, carnes, pescado y mariscos). Aspectos como practicidad (excluyendo ubicación), financiamiento, calidad del lugar o servicios adicionales, difícilmente pueden ser considerados ventajas. Sea porque son mencionados por un reducido número de encuestados, o bien, porque la calificación asignada es apenas satisfactoria, como en el caso de la calidad del lugar.

La suma de los resultados anteriores puso en evidencia que los locatarios identificaban todas las variables que influyen en su competitividad pero que mantenían supuestos equivocados respecto al valor de cada una de ellas, lo que a su vez mostró la necesidad de dotarlos de elementos de juicio para que pudieran priorizarlas en forma correcta.

La evidencia del desconocimiento de las características y los deseos de la clientela fue la clave para propiciar el aprendizaje, lo que comprobó la 
validez del método propuesto (Trabajo-Aprendizaje) ante situaciones tan diferentes a aquéllas en las que había sido aplicado.

Derivado de los ejercicios descritos, se advierte que futuros programas de formación a locatarios de mercados públicos debe contemplar el concepto de calidad que, concretamente, atrae a su clientela y los mecanismos para garantizarla; los elementos a considerar para que su precio mantenga su competitividad; el segmento de mercado que atienden y sus implicaciones, así como herramientas para que sean capaces de valorar la satisfacción del cliente.

En el caso de El Palmar y Las Flores el programa de formación-investigación permitió que los locatarios comprendieran que la reconfiguración de los hábitos de consumo de los clientes no depende de manera exclusiva del surgimiento de nuevos competidores sino de variables determinadas por sus condiciones de vida y trabajo. Este aprendizaje les permitió focalizar mejor a su más fuerte competidor: el tianguis, así como las razones de su posición en el gusto de sus clientes. Y, por tanto, les mostró la necesidad de conocer mejor a su clientela.

Los aprendizajes condujeron, a su vez, a dar continuidad a las sesiones de formación y al proceso de investigación; con respecto a este último, destaca el compromiso de realización de una encuesta entre la población que rodea a los mercados y que acude a los mismos, o lo hace ocasionalmente, esto con el fin de precisar las opiniones de los clientes eventuales o potenciales sobre los mercados y, por otra parte, derivó en el compromiso de elaborar un Plan de Negocios para el acopio y venta de jitomate. Dicho plan fue concebido con la intención de mejorar las ventajas competitivas (en precio y calidad) de uno de los principales ganchos del mercado público: el jitomate. Situación que deberá contribuir a afianzar la posición de los mercados en cuestión entre la población que ya es clientela y que, además, deberá mostrar el camino a seguir a los comerciantes de otros productos para mejorar la combinación calidad/precio y, por ende, sus ingresos. 


\section{Anexo}

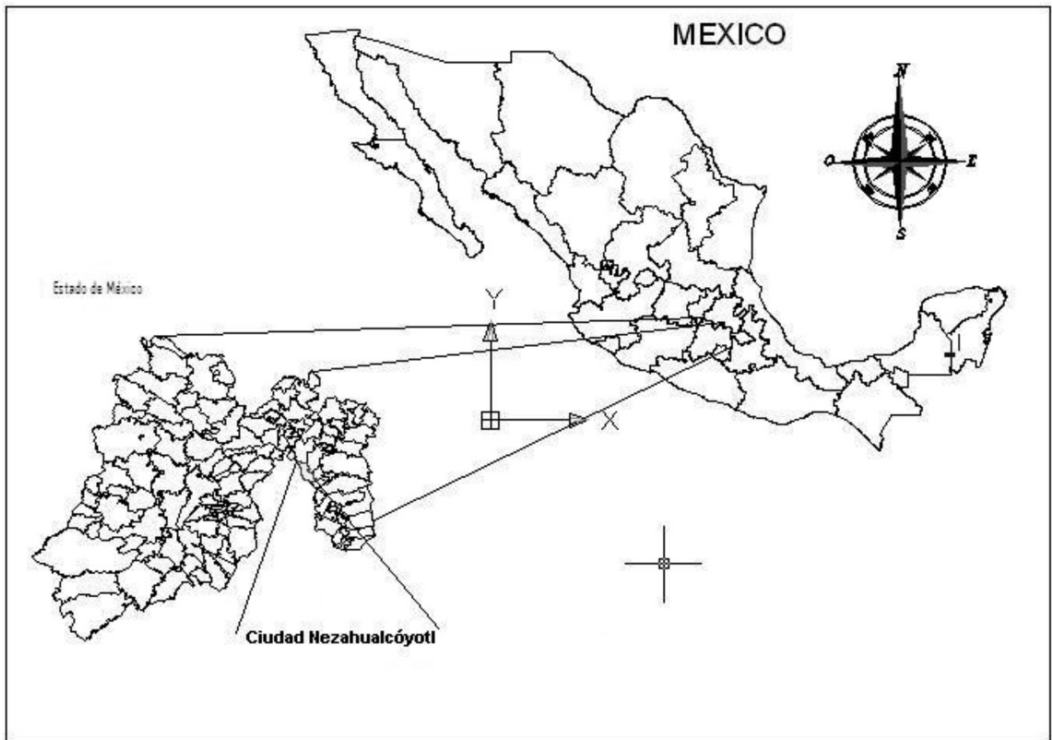

Fuente: Elaboración propia a partir de los mapas del país y del Estado de México.

\section{Bibliografía}

Abud, Rocío y Luz Aydeé González (2009), "Programa de comercio detallista”, Serie Nuestra Experiencia núm. 3, Fundes, México.

ACNielsen (2006), Cambios en el mercado Latinoamericano, http:// mx.nielsen.com/site/documents/esp-mexico, enero de 2009.

Alonso Rivas, Javier e Ildefonso Grande Esteban (2004), Comportamiento del consumidor, EsIC editorial, Madrid.

Bocanegra Gastelum, Carmen O. (2008), El comercio en México y su encuentro con la globalización. El caso Sonora, Universidad de Sonora, Hermosillo.

Bocanegra Gastelum, Carmen O. (2007), "Globalización y comercio: El impacto de Walmart en Sonora”, en Alejandro Álvarez Béjar y Gabriel Mendoza Pichardo (coords.), Impactos regionales, sectoriales y locales en el México del siglo XXI, Itaca-UnAM-Universidad Veracruzana-Universidad de Sonora, México, pp. 177-198. 
Bocanegra Gastelum Carmen O. y Miguel A. Vázquez Ruiz (2003), "Modernización en el comercio detallista", Notas. Revista de información y análisis, 23, INEGI, México, pp. 17-25.

Conapo (Consejo Nacional de Población) (2005), "Proyecciones de la Población de México 2005-2050, Conapo, México, en http:// www.conapo.gob.mx/00cifras/5.htm, diciembre de 2007.

Duch G., Irene, Francoise Garibay y Erick Quesnel G. (2005), La capacitación otra mirada. Lecciones de experiencias mexicanas de capacitación rural, Secretaría de Educación Pública-Universidad Pedagógica Nacional-Organización de las Naciones Unidas para la Educación, la Ciencia y la Cultura-El Colegio de Tlaxcala, A.c.Asociación Nacional de Organismos de Competencia laboral, A.C., México.

Duch G. Irene (2007), "Una alternativa pedagógica para la capacitación”, tesis de licenciatura, Facultad de Filosofía y Letras de la UnAMColegio de Pedagogía, México.

Duhau Emilio y Ángela Giglia (2007), Globalización e informalidad en la ciudad de México. Prácticas de consumo y movilidad, Centro de Estudios Mexicanos y Centroamericanos, México, www.cemca. org.mx/trace/TrACE_5 I_pdf/Duhau_Giglia_51_2007.pdf, septiembre de 2007.

Garza-Bueno, Laura Elena (2006), "Un nuevo paradigma en la formación de recursos humanos como estrategia de desarrollo rural", Revista Agricultura, Sociedad y Desarrollo, 3 (2), Colegio de Postgraduados, Texcoco, pp. 155-173.

GEM (Gobierno del Estado de México) (s/f), "Presentación del Programa de modernización de mercados de venta al detalle", Gobierno del Estado de México, México.

Hernández Luquín, Liliana (2005), “Antecedentes inmediatos de los mercados en Guadalajara", Mercadotecnia global. Revista de mercados y negocios internacionales, año 8, época 1 (50), ITEso, Universidad Jesuita de Guadalajara, México, http://mktglobal.iteso. $\mathrm{mx} /$, octubre de 2007 . 
Hernández Luquín, Liliana (2006a), "Situación Socioeconómica de los mercados públicos de la ZMG frente a los supermercados", Mercadotecnia global. Revista de Mercados y Negocios Internacionales, Año 9, Época 1, (51), ITEso, Universidad Jesuita de Guadalajara, México, http://mktglobal.iteso.mx/, octubre de 2007.

Hernández Luquín, Liliana (2006b), "El consumidor de productos básicos en la Zona Metropolitana de Guadalajara", Mercadotecnia global. Revista de mercados y negocios internacionales, Año 9, Época 1 (52), Iteso, Universidad Jesuita de Guadalajara, México, http://mktglobal.iteso.mx/, octubre de 2007.

INEGI (Instituto Nacional de Estadística, Geografía e Informática) (1999), “Censos económicos 1999”, D.F., INEGI, México.

INEGI (Instituto Nacional de Estadística, Geografía e Informática) (2004), “Censos económicos 2004”, D.F., INEGI, México.

Kinnear, Thomas C. y James R. Taylor (1996), Investigación de mercados. Un enfoque aplicado, McGraw-Hill, México.

Kotler, Philip y Gary Armstrong (1998), Fundamentos de Mercadotecnia, Prentice-Hall Hispanoamericana, s.A., México.

Kotler, Philip y Gary Armstrong (2001), Marketing, Pearson Educación, México.

Mollá Descals, Alejandro (2006), "Estrategia de marketing y comportamiento del consumidor", en Alejandro Mollá Descals (coord.), Gloria Berenguer Contrí, Miguel Ángel Gómez Borja e Ismael Quintanilla Pardo, Comportamiento del consumidor, Editorial Uoc, Barcelona, pp. 13-38.

Porter, Michael E. (1999), Ventaja competitiva. Creación y sostenimiento de un desempeño superior, Compañía Editorial Continental, s.A. de c.v., México.

Reardon, Thomas y Julio Berdegué (2002), “The rapid rise of supermarkets in Latin America: Challenges and oportunities for development", Development Policy Review, 20 (4), Overseas Development Institute, London, pp. 371-388. 
Rivera Camino, Jaime, Rolando Arellano Cueva y Víctor M. Molero Ayala (2000), Conducta del consumidor. Estrategias y tácticas aplicadas al marketing, Esic Editorial, Madrid.

Schwentesius Rindermann, Rita y Manuel Ángel Gómez Cruz (2006), "Supermercados y pequeños productores hortofrutícolas en México", Revista Comercio Exterior, 56 (3), Banco de Comercio Exterior, sNC, México, pp. 205-218.

Secretaría de Economía (2008), Agenda de competitividad para las centrales de abasto 2008-2012. Secretaría de Economía, México, http://www.elogistica.economia.gob.mx/work/models/elogistica/ Resource/1/1/images/ABASTO0812.pdf, diciembre de 2008.

Recibido: 11 de mayo de 2009. Reenviado: 14 de mayo de 2011. Reenviado: 31 de octubre de 2011. Aceptado: 27 de febrero de 2012.

Laura Elena Garza-Bueno. Es licenciada en economía por el IPN, con doctorado de la Universidad Estatal de Moscú. Actualmente es profesora e investigadora del Colegio de Postgraduados y profesora por asignatura en la Universidad Autónoma Chapingo. Desde 1985, año en que ingresó a la academia, ha contribuido a las labores de docencia, investigación y servicio en sus diversas modalidades. Es miembro del Sistema Nacional de Investigadores y participa en la Coordinación de la Maestría Tecnológica en Prestación de Servicios Profesionales impulsada por la Financiera Rural y el Colegio de Postgraduados. En el servicio público ha asumido diversas responsabilidades vinculadas con la formación de recursos humanos. Sus líneas de investigación son: Desarrollo rural, formación de recursos humanos, mujeres y microfinanciamiento. Entre sus últimas publicaciones se encuentran: Conversión del trabajo en el objeto de estudio, Colegio de Postgraduados y Financiera Rural, México (2009); en coautoría, Con el sol a cuestas. Balance de la vejez en el México rural, IndesolGrupo Interdisciplinario sobre Mujer, Trabajo y Pobreza A.c., México (2008); "Un nuevo paradigma en la formación de recursos humanos como estrategia de desarrollo rural", en Revista Agricultura, Sociedad y Desarrollo, 3 (2), Colegio de Postgraduados, Texcoco, pp. 155-173 (2006). 